\title{
Estrategias para la búsqueda de justicia por crímenes internacionales de género: el caso Ríos Montt
}

\section{Strategies for the search of justice in international gender crimes: the Rios Montt case}

\author{
Paloma Soria Montañez \\ Women's Link Worldwide, España
}

Licenciada en Derecho por la Universidad de Málaga y Máster en Acción Solidaria Internacional por la Universidad Carlos III de Madrid. Desde 2006 trabaja en Women's Link Worldwide, donde es abogada senior y coordinadora del programa "Crímenes Internacionales de Género". Como abogada experta en género, ha litigado exitosamente ante tribunales nacionales e internacionales y ha sido requerida como experta en varios casos de genocidio ante tribunales de Guatemala, destacando su participación como perita en el juicio contra Efraín Ríos Montt, así como en Ecuador en procesos por crímenes de lesa humanidad. Es autora y coautora de diversas publicaciones en materia de género y ha impartido conferencias y seminarios de formación a jueces/zas y fiscales en España, Guatemala, Argentina y Marruecos, entre otros.

p.soria@womenslinkworldwide.org

\section{RESUMEN}

La incorporación del enfoque de género en los procesos de justicia por crímenes internacionales es un reto que tienen actualmente los tribunales nacionales. En este sentido, los órganos investigadores y juzgadores, así como los representantes de las víctimas y la sociedad civil en general, tienen el desafío de buscar estrategias que permitan probar los crímenes internacionales de naturaleza sexual, evitando la revictimización de las víctimas sobrevivientes. Este artículo describe la estrategia utilizada para enfrentar crímenes de género en el juicio por genocidio y delitos de deberes contra la humanidad contra Ríos Montt en Guatemala, una experiencia innovadora para visibilizar los aspectos de género involucrados en los crímenes internacionales, en concreto, a través del trabajo con víctimas-sobrevivientes, y del uso del peritaje de género como estrategia legal destinada a evitar o minimizar la re-victimización de los testigos en los procesos que buscan justicia por crímenes internacionales. El artículo concluye que dicho proceso permite determinar algunos de los retos que existen actualmente en la investigación y enjuiciamiento de los crímenes internacionales desde un enfoque de género.

Palabras clave: Violencia sexual - género- crímenes internacionales - peritaje.

\section{ABSTRACT}

Nowadays national Courts face the challenge of considering a gender perspective within international crimes cases. Courts and prosecutors, as well as victim's defendants and civil society, face the challenge of looking for strategies to proof international sex crimes that avoid the re-victimization of survivors. This article describes the strategy used to face gender crimes in Rios Mont case in Guatemala regarding genocide and crimes against humanity. This innovative experience was useful to points out gender aspects involved in international crimes. In practical terms, it refers to the treatment to survivors and the use of gender expert reports conceived as legal strategies to avoid or minimize the re-victimization of eye witnesses in international criminal cases. The article concludes determining some of the challenges that international crimes investigation and judgment currently held from a gender perspective.

Key words: sexual violence - gender - international crimes - expert report. 


\section{Introducción}

En el marco de la justicia internacional, los Tribunales Penales Internacionales para la ex Yugoslavia (TPIY) y Ruanda (TPIR), marcaron un punto de inflexión al judicializar y condenar por primera vez en la historia la comisión de crímenes internacionales utilizando un enfoque de género ${ }^{1}$. Su jurisprudencia ha tenido una influencia decisiva y muy positiva sobre la labor de interpretación de otros tribunales internacionales ${ }^{2}$, así como de tribunales nacionales que, en la actualidad, han asumido también como propio el reto de aplicar una adecuada perspectiva de género al análisis de los crímenes internacionales, no obstante lo reciente de los procesos judiciales nacionales por este tipo de crímenes.

En las jurisdicciones nacionales, la inclusión de una perspectiva de género en estos procesos cuenta con una experiencia breve que ha supuesto un reto y ha requerido la puesta en marcha, por parte de diversos actores sociales y gubernamentales, de estrategias concretas destinadas a no dejar en la impunidad las violaciones de derechos humanos que -en contextos de conflicto armado- impactan de manera exclusiva o desproporcionada a las mujeres y las niñas ${ }^{3}$.

En este escenario, el proceso que condujo al juzgamiento y la condena de Ríos Montt en Guatemala se ha convertido en un referente mundial que merece ser analizado. La sentencia, a pesar de haber sido revocada por un tribunal superior ${ }^{4}$, constituye la primera vez que se condena a un ex jefe de Estado por crímenes de naturaleza sexual y de violencia de género ocurridos bajo su mandato, tales como genocidio y crímenes de guerra ${ }^{5}$. El presente artículo tiene por objeto

CHINKIN, Christine y CHARLESWORTH, Hilary. "Redrawing the boundaries of international law". En: The boundaries of international law. A feminist analysis. Manchester: Manchester University Press, 2000, pp. 308-337; COPELON, Rhonda. "Gender crimes as war crimes: integrating crimes against women into international criminal law". McGill Law Journal, Vol.46, No.3, 2000 y ASKIN, Kelly. "Prosecuting wartime rape and other gender-related crimes under international law: extraordinary advances, enduring obstacles". Berkeley Journal of International Law, No. $21,2003$.

2 DE BROUWER, Anne-Marie. Supranational Criminal Prosecution of Sexual Violence. The ICC and the Practice of the ICTY and the ICTR. Oxford: Intersentia, 2005, p. 20-21; KOENING, Alexa, LINCOLN, Ryany y GROTH, Lauren. The jurisprudence of Sexual Violence. Working Paper Series. Berkeley: Human Rights Center University of Berkeley, 2011. Disponible [en línea] <http://www.law.berkeley.edu/HRCweb/pdfs/SVA_Jurisprudence.pdf> [consulta: 24 marzo 2014] y; OOSTERVELD, Valerie. "The Gender Jurisprudence of the Special Court for Sierra Leone: Progress in the Revolutionary United Front Judgments". Cornell International LawJournal, No. 44, 2011, pp.49-74.

3 Entre las experiencias nacionales, junto con la que se analiza en el presente artículo, resulta relevante tener en cuenta los avances promovidos en la justicia argentina, en el marco de las causas que juzgan los hechos de violencia ocurridos en dicho país entre 1976 y 1983 durante el terrorismo de Estado. En este contexto, en junio de 2010 se emitió la primera sentencia condenatoria por un crimen de género. En concreto, el Tribunal Oral Federal dictó sentencia en el asunto Gregorio Rafael Molina, ex Jefe de la Base Aérea de Mar del Plata, en la que se le condenaba por los actos de violación que ejecutó contra mujeres detenidas en el centro clandestino de detención donde desempeñó su cargo, calificándolos como crímenes de lesa humanidad. Para más información sobre el juzgamiento de hechos de violencia sexual en estos procesos, consultar: Procuración General de la Nación. Informe de gestión de la unidad Fiscal de Coordinación y Seguimiento de las causas por violaciones a los derechos humanos cometidas durante el terrorismo de Estado. Argentina, 2012. Disponible [en línea] <http://www.mpf.gov.ar/docs/ Links/DDHH/Informe_Anual_2012_DDHH.pdf> [consulta: 24 marzo 2014].

Con fecha 20 de mayo de 2013, la Corte Constitucional de Guatemala emitió resolución que ordenaba anular "[...] todo lo actuado en la fase de debate oral y público del proceso subyacente al proceso de amparo [es decir, al proceso existente contra Ríos Montt] a partir del 19 de abril de 2013, quedando anulado todo lo actuado en ese proceso penal con posterioridad a esa fecha". Al momento de emitir esta resolución, Ríos Montt ya había sido condenado por la Corte de instancia, en concreto el Tribunal A de Mayor Riesgo de Guatemala. Como consecuencia de dicha resolución emitida por la Corte Constitucional, así como otras emitidas en el marco del proceso por distintas instancias, actualmente existe una situación de inseguridad jurídica pues no ha quedado claramente determinado si la resolución que condenó a Ríos Montt está anulada, por lo que éste se encuentra actualmente bajo arresto domiciliario.

"El ex dictador Ríos Montt, primer ex jefe de Estado centroamericano condenado por genocidio". El Confiden- 
relatar y analizar parte de la estrategia seguida en el caso Ríos Montt para conseguir que los crímenes de género fueran investigados y condenados, principalmente a través del uso de peritajes de género como instrumento probatorio en los procesos de justicia, visibilizando su utilidad para evitar la revictimización de las personas sobrevivientes de crímenes internacionales de naturaleza sexual. Esperamos que el análisis de la experiencia y de las lecciones aprendidas pueda ser de utilidad para otros procesos similares que se llevan actualmente en la región.

\section{Violencia contra mujeres y niñas durante el conflicto armado interno en Guatemala}

En Guatemala, entre 1962 y 1996 hubo un conflicto armado interno (CAI) que se saldó con la muerte de aproximadamente 200.000 personas y 40.000 desaparecidas $^{6}$. En el marco de los Acuerdos de Paz, se determinó la creación de la Comisión de Esclarecimiento Histórico ${ }^{7}(\mathrm{CEH})$, cuyo mandato sería el esclarecimiento de las violaciones de los derechos humanos y hechos de violencia ocurridos en Guatemala entre enero de 1962 hasta la adopción del Acuerdo de Paz Firme y Duradera de 29 de diciembre de $1996^{8}$.

En el CAI de Guatemala, hombres, mujeres, niños y niñas, sufrieron distintas formas de violencia. Durante 34 años numerosas personas fueron torturadas y sometidas a tratos crueles, inhumanos y degradantes, ejecutadas extrajudicialmente y desaparecidas. Muchas debieron huir a la montaña en un intento por salvar sus vidas, aunque fueron igualmente perseguidas y asesinadas o fallecieron por las condiciones de vida infrahumanas a las que se vieron sometidas. La CEH registró un total de 42.275 víctimas y documentó 7.517 casos de violaciones de derechos humanos $^{9}$.

Otra de las formas de violencia recogidas en la CEH fue la violación sexual contra mujeres y niñas. Se registraron $1.465 \operatorname{casos}^{10}$, lo que constituye un $2 \%$ del total de violaciones documentadas $^{11}$. Este tipo de violencia se ejerció contra mujeres mayas en el $88.7 \%$ de los $\operatorname{casos}^{12}$. El $62 \%$ fueron víctimas adultas, entre los 18 y los 60 años; el 35\% niñas, entre los 0 y los 17 años y; un $3 \%$ correspondía a mujeres ancianas ${ }^{13}$.

La CEH realizó un importante trabajo en la documentación de los hechos ocurridos durante el conflicto, constituyendo un aporte único para la construcción de la verdad en Guatemala. No obstante, el género -como categoría de análisis- no fue incluido a priori, sino que este aspecto fue surgiendo en el proceso de recopilación de testimonios ${ }^{14}$, lo que desembocó en la inclusión

cial. 11 de mayo de 2013. Disponible [en línea] <http://www.elconfidencial.com/mundo/2013/05/11/el-exdictador-rios-montt-primer-exjefe-de-estado-centroamericano-condenado-por-genocidio-120701> [consulta: 24 marzo 2014]; "Ríos Montt: de mandatario a culpable de genocidio". BBC. 10 de mayo de 2013. Disponible [en línea] <http://www.bbc.co.uk/mundo/noticias/2013/05/130510_guatemala_semblanza_rios_montt_jcps.shtml> [consulta: 24 marzo 2014].

CEH. Guatemala: Memoria del silencio. Guatemala, 1999, párr. 212.

Acuerdo sobre el establecimiento de la Comisión para el Esclarecimiento Histórico de las violaciones a los derechos humanos y los hechos de violencia que han causado sufrimientos a la población guatemalteca. Oslo, Noruega, 23 de junio de 1994.

CEH. Guatemala: Memoria del silencio...op. cit, párrs. 61 y 79.

Ibídem, párr.1724.

Ibídem, párr. 2388.

Ibídem, párr.1732.

Ibídem, párr. 2390.

Ibídem, párr. 2391.

PATTERSON-MARKOWITZ, Rebecca, OGLESBY, Elizabeth y MARSTON, Sallie. "Subjects of Change: Feminist 
en el informe final, de un capítulo titulado "Violencia sexual contra la mujer"15.

La falta de una adecuada metodología hizo que los hechos de violencia sexual contra hombres no fueran documentados, y que se documentaran principalmente hechos de violación, mientras que otros actos de violencia sexual, tales como mutilaciones, esclavitud sexual en destacamentos o uniones forzadas, no fueran objeto de estudio pormenorizado.

\section{Crímenes internacionales de género: estrategias para su persecución en el caso Ríos Montt}

En el año 2001, la Asociación para la Justicia y la Reconciliación (AJR) $)^{16}$ y el Centro para la Acción Legal en Derechos Humanos $(\mathrm{CALDH})^{17}$, presentaron una denuncia ante el Ministerio Público de Guatemala por genocidio y delitos contra los deberes de humanidad ${ }^{18}$ contra Efraín Ríos Montt, por los crímenes cometidos mientras actuó como jefe de Estado entre el 23 de marzo de 1982 y el 8 de agosto de 1983, en el marco de la política militar de "tierra arrasada"19.

En este proceso, las organizaciones de la sociedad civil en conjunto con el Ministerio Público, lograron incluir en los procesos judiciales el análisis de la violencia sexual contra las mujeres y niñas. Esto fue posible gracias a los testimonios de diez mujeres que declararon en el juicio oral, junto con el peritaje de género presentado y ratificado durante el juicio.

\subsection{Acompañamiento a las víctimas-testigos}

El trabajo con personas sobrevivientes para su comparecencia como testigos en procesos de justicia por crímenes internacionales, resulta difícil e impone una serie de desafíos, especialmente en los casos de violencia sexual. Así, por un lado, los equipos que trabajan en la estrategia legal del caso tienen el interés de probar los elementos del crimen, lo que les obliga a tratar de obtener la mayor cantidad de información y datos por parte de las víctimas y; por el otro, este trabajo debe ser compatible con las necesidades de las víctimas y sus procesos de sanación y superación del trauma ${ }^{20}$. Lograr esta compatibilidad entre necesidades y objetivos procesales fue el primer reto que tuvieron que superar las partes implicadas en el proceso judicial contra Ríos Montt, que tenía por objeto demostrar el uso de la violencia sexual contra las mujeres y niñas mayas ixiles como un acto para la destrucción del grupo por parte del Estado.

Geopolitics and Gendered Truth-Telling in Guatemala". Journal of International Women's Studies, No.13, 2012, p.88.

bídem, p. 89

Para más información sobre la organización, visitar: [en línea] <www.ajrguatemala.org/> [consulta: 24 de marzo de 2014]. op. cit, párrs. 787-793.

Para más información sobre la organización, visitar: [en línea] <www.caldh.org>[consulta: 24 de marzo de 2014].

Los crímenes de genocidio y de delito contra los deberes de humanidad están consagrados, respectivamente, en los artículos 376 y 378 del Código Penal de Guatemala.

Para una descripción de la política militar de "tierra arrasada" consultar: CEH. Guatemala: Memoria del silencio...

Para observar estudios que analizan los proyectos que tienen como objetivo el desarrollo de procesos de sanación de mujeres que han sufrido violencia sexual en Guatemala y los procesos de justicia, ver: Equipo de Estudios Comunitarios, Acción psicosocial y Unión Nacional de Mujeres Guatemaltecas. Tejidos que lleva el alma. Memoria de las mujeres mayas sobrevivientes de violación sexual durante el conflicto armado. Guatemala: ECAP, UNAMG, 2009. Para conocer experiencias comparadas de distintos países de América Latina en trabajos de acompañamiento psicosocial a mujeres sobrevivientes de violencia sexual y procesos de justicia, ver: Consejería en Proyectos y PCS Guatemala. Compartir la memoria colectiva. Acompañamiento psicosocial y justicia integral para mujeres víctimas de violencia sexual en conflictos armados. Guatemala: PCS, 2007. Para ahondar en el análisis del acompañamiento psicosocial en procesos de justicia, ver: MARTíN, Carlos. Acompañar los procesos con las víctimas: atención psicosocial en las violaciones de derechos humanos. Colombia: PNUD, 2012, p. 74-83. 
Para lograrlo, se desarrolló un trabajo de acompañamiento a las mujeres en sus procesos de sanación, que se prolongó durante años y se mantiene hasta la actualidad. AJR y CALDH acudieron a las comunidades a informar a las mujeres sobre el caso que se había presentado y su importancia, además les preguntaron si querían ser partícipes del mismo. Tras estas visitas se realizaron talleres de sanación con las mujeres interesadas, quienes paulatinamente consiguieron crear una relación de confianza que culminó en la presentación de los testimonios relatados durante la causa.

Durante los encuentros con las mujeres que participaron en el proceso, tuvimos la oportunidad de hablar con ellas de lo ocurrido durante el conflicto y conocer todas las formas de violencia de las que fueron víctimas, con lo que logramos entender el significado e impacto que generó en ellas la violencia, en su calidad de mujeres indígenas.

Entre otros, los esfuerzos realizados tenían el fin de transmitirles a las mujeres indígenas la importancia de denunciar la violencia que habían vivido, incluida la sexual, y hacerlas sentirse parte del proceso de búsqueda de justicia. Así, este trabajo conjunto estuvo orientado a que las mujeres se "apropiaran" del proceso judicial y lo asumieran como una oportunidad para dar a conocer lo que había pasado en Guatemala y compartir su experiencia que, si bien es propia, también refleja la realidad de muchas otras mujeres alrededor del mundo.

Esta labor que las organizaciones litigantes desarrollaron con las mujeres, permitió que diez de ellas testificaran ante el tribunal y dieran a conocer la realidad que vivieron durante el CAI.

Así, el 2 de abril de 2013 diez mujeres indígenas mayas ixiles testificaron ante el Tribunal A de Mayor Riesgo de Guatemala en el proceso contra Ríos Montt y relataron los actos de violencia que habían sufrido ${ }^{21}$. Estas diez mujeres tuvieron la valentía de sentarse frente a quienes las deshumanizaron e intentaron exterminar su esencia, sus cuerpos y sus vivencias, consiguiendo explicar con sus palabras, al Tribunal y al mundo entero, lo que es el horror, relatando las más extremas formas de crueldad a las que fueron sometidas durante el conflicto.

Recogiendo el testimonio de estas diez valientes mujeres, la sentencia ${ }^{22}$ determinó que los testimonios "demuestran en forma contundente que sí existió violación de mujeres por parte de soldados del Ejército de Guatemala" y que aún hoy es evidente el dolor que experimentan al recordar los hechos consecuencia de la violencia física y psicológica que sufrieron ${ }^{23}$.

21 "Mujeres narran violaciones en juicio a Ríos Montt". Prensa Libre. 2 de abril de 2013. Disponible [en línea] <http:// www.prensalibre.com/noticias/justicia/Mujeres-narran-violaciones-juicio-Rios_Montt_0_893910877.html>[consulta: 11 febrero 2014]; "Escalofriantes relatos de mujeres ixiles en juicio contra Ríos Montt". El Periódico. 2 de abril de 2013. Disponible [en línea] <http://www.elperiodico.com.gt/es/20130402/pais/226557/> [consulta: 11 febrero 2014]; "Víctimas de violaciones masivas testifican en el juicio a Ríos Montt". El País. 2 de abril de 2013. Disponible [en línea] <http://internacional.elpais.com/internacional/2013/04/01/actualidad/1364772276_557699. html> [consulta: 11 febrero 2014].

Tribunal Primero de Sentencia Penal Mayor Riesgo "A" de Guatemala. Sentencia de 10 de mayo de 2013. C-010762001-00015 OF. $2^{\circ}$.

23 Ibídem, pp.688-689. En relación con las afectaciones generadas por la violencia sexual, ver también: Corte IDH. Caso Masacre Plan de Sánchez vs. Guatemala. Sentencia de 19 de noviembre de 2004. Serie C No. 116, párr. 49.19: "Las mujeres que fueron objeto de violencia sexual por parte de agentes del Estado el día de la masacre y que sobrevivieron a la misma, continúan padeciendo sufrimientos por dicha agresión. La violación sexual de las mujeres fue una práctica del Estado, ejecutada en el contexto de las masacres, dirigida a destruir la dignidad de la mujer a nivel cultural, social, familiar e individual. Estas mujeres se perciben como estigmatizadas en sus comunidades y han sufrido por la presencia de los victimarios en las áreas comunes del municipio. Además, la impunidad en la que permanecen estos hechos ha impedido que las mujeres participen en los procesos de justicia". 
Esta experiencia demuestra lo difícil que resulta para las víctimas-sobrevivientes articular su propio sufrimiento y expresarlo públicamente, lo cual revela los grandes retos que enfrentan la administración de justicia y las organizaciones que representan a las víctimas. Trabajar desde un enfoque que priorice las necesidades de la víctima por sobre las del proceso penal, requiere poner a disposición de estas todos los recursos necesarios para su protección física y emocional, creando un entorno de seguridad que haga posible el testimonio. Esto es muy arduo de lograr tomando en cuenta las características de los procesos penales y la naturaleza inquisitorial de los interrogatorios. Por tanto, este es el reto más importante que tenemos por delante y la experiencia en Guatemala puede servir como inicio para la reflexión y construcción de buenas prácticas $^{24}$.

\subsection{El peritaje como herramienta de apoyo a los testimonios}

Junto con los testimonios de las mujeres sobre violencia sexual, durante el proceso judicial contra Ríos Montt se realizó la presentación de un peritaje sobre crímenes de género.

El antecedente del uso de peritajes de género como estrategia se sitúa en el año 2008, cuando como abogada de "Women's Link Worldwide" ${ }^{25}$ comencé a trabajar en conjunto con el "Center for Justice and Accountability"26, en el caso que estaba investigándose en la Audiencia Nacional de España por los crímenes de terrorismo, genocidio y torturas ocurridos en Guatemala durante el CAI, en base al principio de justicia universal. El objetivo del trabajo fue visibilizar e incluir los crímenes de género en la investigación. Teníamos por delante un caso que desde finales de 2005 había conseguido presentar ante la audiencia alrededor de 40 testimonios, entre víctimas-sobrevivientes y peritos/as, pero apenas habían surgido los hechos de violencia sexual durante el proceso. Por tanto, había que conseguir demostrar por qué los crímenes de género debían ser investigados, juzgados y sancionados en el marco de la causa, evitando que víctimas-sobrevivientes volvieran a declarar para preguntarles sobre actos de violencia sexual, para prevenir una posible re-victimización.

Tras un proceso de análisis y discusión acerca de las distintas vías legales existentes para lograr este objetivo, se decidió presentar una ampliación de querella y junto con esta, solicitar que se admitieran dos peritajes que aportaran, por un lado, el análisis normativo que de acuerdo al Derecho Penal Internacional establece la obligatoriedad de investigar los crímenes de género y, por otro, un análisis pormenorizado de la violencia contra mujeres y niñas ocurrida durante el conflicto $^{27}$. Esta estrategia legal ${ }^{28}$ resultó exitosa y terminó con la emisión de un auto de fecha 26 de julio de 2011, el cual afirmaba que:

24 Es muy importante destacar la labor de numerosas organizaciones de mujeres que llevan años trabajando y promoviendo procesos de sanación, empoderamiento y justicia con víctimas-sobrevivientes de violencia sexual durante el CAI. Para información al respecto, consultar, entre otros: PATTERSON-MARKOWITZ, Rebecca, OGLESBY, Elizabeth y MARSTON, Sallie. Subjects of Change... op.cit, pp. 10-14 y AGUILAR, Yolanda. La violencia sexual durante el conflicto armado interno en Guatemala y la necesidad de recordar para construir memoria colectiva desde las mujeres. Ponencia presentada en 1er Encuentro de Salud Mental en Guatemala, 2005. Disponible [en línea] <http://www.nodo50.org/mugarik/files/Yolanda\%20Aguilar.pdf> [consulta: 25 de marzo de 2014].

Para más información sobre la organización, visitar: [en línea] <www.womenslinkworldwide.org> [consulta: 24 de marzo de 2014].

26 Para más información sobre la organización, visitar: [en línea] <www.cja.org> [consulta: 24 de marzo de 2014 ].

27 Los peritajes fueron presentados por Patricia Viseur Sellers, ex fiscal y asesora legal en género para los Tribunales Penales Internacionales y María Eugenia Solís, abogada y notaria experta en género y niñez. 
[...] los delitos de genocidio, terrorismo, lesa humanidad, torturas, asesinato y detenciones ilegales [...] es necesario (en aras a cumplir satisfactoriamente con el deber de investigar, juzgar y sancionar estos graves crímenes internacionales que recae en este Juzgado) considerarlos también como crímenes de género: las conductas criminales fueron generalizadas y sistemáticas y pretendían acabar con la población maya, no solo a través del exterminio físico sino también del quebrantamiento de la estructura social, por lo que significaba atentar contra las mujeres mayas $^{29}$.

Quedaban así incluidos en la investigación los crímenes de género y sentado un precedente que no tardó en trasladarse a Guatemala. Así, apenas un mes después, en agosto de 2011, presenté, a instancias del Ministerio Público y de CALDH, el peritaje "La violencia contra la mujer indígena maya ixil en el conflicto interno en Guatemala" en el marco del proceso existente contra Ríos Montt. El peritaje exponía, en primer término, la configuración de los crímenes internacionales desde un enfoque de género en la normativa y la jurisprudencia de acuerdo con el Derecho Penal Internacional, el Derecho Internacional Humanitario y el Derecho Internacional de los Derechos Humanos ${ }^{30}$. Teniendo en consideración que era el primer caso en el que se juzgaba por el crimen de genocidio en Guatemala y toda América Latina, no existía precedente jurisprudencial alguno en el que apoyarse. Por ello, el peritaje pretendía ser un respaldo legal para el tribunal, al recoger la jurisprudencia emanada de tribunales internacionales.

Posteriormente, el peritaje analizaba las formas de violencia de género ocurridas en el contexto del CAI de Guatemala, para determinar la configuración de crímenes internacionales. De esta manera, el peritaje tenía como objeto ser respaldo de los testimonios que existían en el expediente de la causa, otorgando validez y legitimidad a los mismos. Con independencia del contexto en donde ocurran este tipo de delitos, los procesos por violencia sexual conllevan un riesgo emocional muy alto para las supervivientes que frecuentemente ven cuestionada su credibilidad, aumentando sus sentimientos de culpa y vergüenza. En este sentido, el peritaje constituye una forma de validar la experiencia individual en casos de violencia sexual, evitando una mayor revictimización, al mismo tiempo que fortalecen la calidad probatoria del testimonio de la víctima.

Igualmente, resultaba de gran importancia que el peritaje demostrara que la violencia sexual en el marco de los conflictos armados comprende actos que van más allá de la violación, y que tanto mujeres como hombres pueden ser víctimas de esa violencia. En ese sentido, el peritaje explicaba por qué debía incluirse un enfoque de género en el análisis de la violencia, consistente en analizar las distintas formas de violencia que se cometieron contra hombres y mujeres en el conflicto, así como su distinto significado y fin. Es cierto que la aplicación práctica de este enfoque resultaba compleja en el caso de Guatemala, porque el informe de la CEH se constitu-

29 Para acceder al auto completo visitar: [en línea] <http://www.womenslinkworldwide.org/wlw/new.php?modo=detalle_proyectos\&tp=proyectos\&dc=22> [consulta: 24 de marzo de 2014].

Entre los precedentes citados en el peritaje, destacan: TPIR. Prosecutor v. Jean Paul Akayesu. Caso No. ICTR-964-T, 2 de septiembre de 1998; TPIY. Prosecutor v. Furundzija. Caso No. IT-95-17/1-T, 10 de diciembre de 1998; TPIY. Prosecutor v. Kunarac et al. Caso No. IT-96-23-T, 22 de febrero de 2001; TPIY. Prosecutor v. Zejnil Delalic et al. Caso No. IT-96-21-T, 16 de noviembre de 1998; TPIY. Prosecutor v. Krstic. Caso No. IT-98-33, 2 de agosto de 2001; TPIY. Prosecutor v. Stakic. Caso No. IT-97-24-T, 31 de julio de 2003; Tribunal Especial para Sierra Leona. Prosecutor v. Issa Hassan Sesay, Morris Kallon y Augustine Gbao. Caso No. SCSL-04-15-T, 2 de marzo de 2009; Tribunal Especial para Sierra Leona. Prosecutor v. Brima, Kamara y Kanu. Caso No. SCSL-04-16-T, 22 de febrero de 2008; Corte Penal Internacional. Prosecutor v. Germain Katanga and Mathieu Ngudjolo Chui. Caso No. ICC01/04-01/07, 30 de septiembre de 2008; Corte IDH. Caso González y otras ("Campo Algodonero") vs. México. Excepción Preliminar, Reparaciones y Costas. Sentencia de 16 de noviembre de 2009. Serie C No. 205; Corte IDH. Caso Rosendo Cantú y otra vs. México. Excepción Preliminar, Fondo, Reparaciones y Costas. Sentencia de 31 de agosto de 2010. Serie C No. 216; Corte IDH. Caso de la "Masacre de Mapiripán" vs. Colombia. Sentencia de 15 de septiembre de 2005. Serie C No. 134. 
yó como una de las pruebas documentales de referencia, y en el mismo solo se documentaron actos de violencia sexual contra mujeres y niñas. No obstante, era importante dejar constancia de que la investigación y enjuiciamiento de estos crímenes siempre debe comprender una indagación sobre el impacto y formas de violencia diferenciadas en razón del género.

El proceso que llevó hasta la presentación el peritaje enfrentó numerosos obstáculos en el tiempo, hasta que en enero de 2013, el Juez Primero B de Mayor Riesgo, Miguel Ángel Gálvez, dio apertura de juicio contra José Efraín Ríos Montt y José Mauricio Rodríguez Sánchez, por los delitos de genocidio y crímenes de lesa humanidad. Así, el 19 de marzo de 2013 comenzó el juicio oral contra ambos ${ }^{31}$ y el 12 de abril participé en el debate oral para ratificar el peritaje y realizar una breve exposición del mismo ${ }^{32}$.

En el debate oral, junto con los principales asuntos tratados en el peritaje escrito, el reto principal fue mostrar y transmitir el mensaje de que los distintos actos de violencia sexual que ocurrieron en Guatemala se habían ejecutado con la intención de destruir al grupo indígena maya ixil, y no habían sido "excesos" producidos en el marco del CAI, ni daños colaterales, sino crímenes tan graves como las ejecuciones o las desapariciones. Por eso era importante evidenciar cómo las normas de derecho internacional así como la jurisprudencia emanada de diversos tribunales internacionales, afirman que dichos actos podían ser constitutivos de crímenes internacionales.

Asimismo, era fundamental transmitir que el concepto "destrucción" va más allá de lo físico ${ }^{33}$. Por ello, fue importante analizar el significado que la sexualidad tiene dentro de la cultura maya, de tal manera que se entendiera por qué el uso de la violencia sexual podía constituir una vía para la destrucción del grupo no sólo física, sino también de la cultura y el tejido social.

El resultado final de esta estrategia combinada, que construyó un vínculo entre los testimonios y el peritaje especializado en género, fue un hecho histórico cuando el 10 de mayo el Tribunal A de Mayor Riesgo de la Ciudad de Guatemala condenó a 80 años de prisión a Efraín Ríos Montt por genocidio y crímenes de guerra, y la sentencia incluyó la condena de los crímenes de género. Dicha sentencia asumió claramente tanto los testimonios de las mujeres ixiles como el peritaje sobre crímenes internacionales de género. La sentencia afirma que las mujeres ixiles sufrieron violencia sexual:

[...] para lograr la ruptura del tejido social y lograr la eliminación de la semilla ixil, siendo por lo tanto los actos de violencia sexual y métodos usados, formas de destruir al grupo, comprobándose así la intención de destruir al grupo completo. Siendo las violaciones sexuales a las mujeres prueba objetiva de la intención de destruir al grupo ${ }^{34}$.

31 FIDH. Genocidio en Guatemala: Ríos Montt culpable, No. 6, 2013.

32 "Jurista: la violación se utilizó de castigo". Siglo XXI. 13 de abril de 2013. Disponible [en línea] <http://www.s21. com.gt/node/299858> [consulta: 11 febrero 2014] y "Peritos señalan secuelas imborrables de la guerra". El Periódico. 13 de abril de 2013. Disponible [en línea] <http://www.elperiodico.com.gt/es/20130413/pais/226892/> [consulta: 11 febrero 2014].

33 Entre otras fuentes, para la interpretación del concepto de destrucción, el peritaje recogió la siguiente sentencia: TPIR. Prosecutor v. Jean Paul Akayesu, op.cit : "[...]violación y violencia sexual[...]constituyen genocidio en la misma medida que cualquier otro acto siempre y cuando fueran cometidos con la intención específica de destruir, total o parcialmente, a un grupo en particular, y seleccionado como tal [...][l]a violencia sexual fue una parte integral del proceso de destrucción, específicamente dirigido a las mujeres tutsi y contribuyó particularmente a su destrucción y a la destrucción de los tutsi como grupo[...] - destrucción de su espíritu, del deseo de vivir, y de la vida misma", párrs. 731-732.

Tribunal Primero de Sentencia Penal de Mayor Riesgo "A" de Guatemala, op.cit, p.689. 
Asimismo, señala que la mujer fue objetivo de guerra, concluyendo que:

[...] a las mujeres embarazadas se les sacó el niño porque 'es una semilla que hay que matar', circunstancia que apreciamos los juzgadores porque evidencia en forma objetiva la intención de hacer desaparecer al grupo maya ixil, buscando romper con la figura de la mujer, porque es portadora de vida la que transmite los valores de la comunidad, la que da los conocimientos básicos para la vida, siendo admirable para los jueces la forma en que los ixiles han defendido su identidad cultural aún y en medio de la adversidad, porque es claro que se prohibió hablar el idioma materno en cual han conservado como elemento cultural de resistencia a la imposición cultural y militar a la que fueron sometidos ${ }^{35}$.

Finalmente, la sentencia asume el peritaje de género e indica que las afirmaciones realizadas sobre la violencia contra las mujeres se confirman:

\begin{abstract}
[...] con el peritaje efectuado por la perito Paloma Soria Montañez, quien fue precisa en acreditar en su peritaje que el ataque contra las mujeres fue sistemático, siendo parte de una estrategia para destruir a los ixiles. Ataque que incluye violación de menores, mujeres embarazadas y ancianas. El peritaje efectuado constata que las violaciones se produjeron en forma continuada y contribuyeron a la destrucción del tejido social, explicando que tanto las violaciones como las mutilaciones ocasionaron trauma de terror destrucción física y cultural teniendo como objetivo eliminar a la etnia maya ixil ${ }^{36}$.
\end{abstract}

En base a estas pruebas, la sentencia condenó los hechos de violencia contra las mujeres como genocidio y crímenes de guerra.

\title{
3. Mirando al futuro: retos en el trabajo con enfoque de género en los juicios por crímenes internacionales
}

En el caso de Guatemala, los hechos relatados en el juicio y asumidos en la sentencia pasan a constituir verdad, en la medida que aportan una relectura de la historia oficial del país. Dentro de esta relectura, la realidad vivida por mujeres y niñas y las violencias sufridas por estas, pasan a situarse en un mismo plano que los demás abusos identificados. De esta forma, se toma conciencia de que la esclavitud sexual, las uniones forzadas, la violación, los efectos del desplazamiento desde un enfoque de género y tantos otros actos violentos que son cometidos de manera exclusiva o exacerbada contra mujeres y niñas, son crímenes tan graves y atroces como las ejecuciones y torturas que ellas mismas padecieron junto a los hombres mayas. Así, la justicia formal ha permitido contar la verdad de mujeres y niñas, generando una ruptura del silencio que ha mostrado que la verdad individual de muchas mujeres es también una verdad colectiva. Todo ello supone un avance único para los procesos de reparación, ya que permite a las víctimas afrontar el sufrimiento, la culpa y la vergüenza desde la restitución pública de su dignidad. La sentencia contra Ríos Montt constituye un hito en la lucha contra la impunidad respecto de los crímenes que viven mujeres y niñas en los conflictos y guerras. Esta sentencia ha conseguido visibilizar en Guatemala y en el mundo entero las atrocidades que afectan a las mujeres, los impactos de estas y la perpetuidad de sus secuelas en el tiempo.

Uno de los retos que se aprecian tras esta experiencia, es que las estrategias de justicia deben ir acompañadas de procesos de sanación de las víctimas-sobrevivientes. En este sentido, resulta importante que los equipos jurídicos integren el enfoque de género en todo el proceso, de manera que las víctimas directas de la violencia no sean las únicas que testifiquen por los hechos

\footnotetext{
35

Ibídem, p.691.

Ibídem, p.689.
} 
sufridos. Así, los distintos peritajes deben también aplicar tal enfoque. En concreto, el uso de peritajes de género se configura como una importante herramienta en estos procesos, pues aportan los argumentos jurídicos que explican por qué determinadas conductas constituyen crímenes internacionales y abordan cuestiones que permiten que la carga de probar tales crímenes no recaiga exclusivamente en las víctimas directas de los mismos. Esto último se convierte en un método que permite evitar, o cuanto menos minimizar, la revictimización de quienes sufren actos de violencia de género en conflictos.

Actualmente el desafío es maximizar el impacto de los procesos de justicia formal. El proceso vivido en Guatemala durante las distintas etapas de la causa contra Ríos Montt puede ser un ejemplo para futuros casos que se presenten ante tribunales guatemaltecos y en toda la región. Asimismo, es una oportunidad para abrir nuevos diálogos en familias, comunidades y la sociedad en general, de manera que se tome conciencia del impacto de los crímenes de género y de la necesidad de luchar contra los prejuicios para no culpabilizar a quienes los sufren, y para la construcción de un proceso de diálogo sobre la necesidad de acabar con la discriminación y la violencia de género también en tiempos de paz.

Recibido: 4 de noviembre de 2013

Aceptado: 28 de febrero de 2014 